\title{
Study on Fault Diagnosis of Rolling Bearing Based on Time-Frequency Generalized Dimension
}

\author{
Yu Yuan, ${ }^{1,2}$ Xing Zhao, ${ }^{2}$ Jiyou Fei, ${ }^{2}$ Yulong Zhao, ${ }^{1}$ and Jiahui Wang ${ }^{2}$ \\ ${ }^{1}$ State Key Laboratory for Manufacturing Systems Engineering, Xian Jiaotong University, Xian 710054, China \\ ${ }^{2}$ School of EMU Application \& Maintenance Engineering, Dalian Jiaotong University, Dalian 116028, China \\ Correspondence should be addressed to Xing Zhao; zhaoxing428@163.com
}

Received 27 August 2014; Accepted 13 November 2014

Academic Editor: Jiawei Xiang

Copyright (c) $2015 \mathrm{Yu}$ Yuan et al. This is an open access article distributed under the Creative Commons Attribution License, which permits unrestricted use, distribution, and reproduction in any medium, provided the original work is properly cited.

\begin{abstract}
The condition monitoring technology and fault diagnosis technology of mechanical equipment played an important role in the modern engineering. Rolling bearing is the most common component of mechanical equipment which sustains and transfers the load. Therefore, fault diagnosis of rolling bearings has great significance. Fractal theory provides an effective method to describe the complexity and irregularity of the vibration signals of rolling bearings. In this paper a novel multifractal fault diagnosis approach based on time-frequency domain signals was proposed. The method and numerical algorithm of Multi-fractal analysis in timefrequency domain were provided. According to grid type $J$ and order parameter $q$ in algorithm, the value range of $J$ and the cut-off condition of $q$ were optimized based on the effect on the dimension calculation. Simulation experiments demonstrated that the effective signal identification could be complete by multifractal method in time-frequency domain, which is related to the factors such as signal energy and distribution. And the further fault diagnosis experiments of bearings showed that the multifractal method in time-frequency domain can complete the fault diagnosis, such as the fault judgment and fault types. And the fault detection can be done in the early stage of fault. Therefore, the multifractal method in time-frequency domain used in fault diagnosis of bearing is a practicable method.
\end{abstract}

\section{Introduction}

Recently, modern industry is gradually developing in the direction of large-scale, continuous, high speed, and artificial intelligence, with the main advantage of improving productivity, reducing the rejection rate, and ensuring quality of products. But on the other hand, once there is some fault happening on modern sophisticated equipment or structure, the maintenance costs would be much increased and may even lead to major accident [1].

Rolling bearings have been widely used in various rotating machinery and play an important role in rotating machinery, which is easy to go wrong. With the improvement of automation equipment and device complexity, as well as the wide usage of large-scale rotating machinery in engineering, high security and advanced fault prediction capability for the devices and the new fault diagnosis methods are required. Therefore, the fault diagnosis analysis of rolling bearing, especially the correct detection of the early failure has practical value in extending service life and reducing cost. There is a wide range of needs in the exploration and application of bearing fault diagnostic. It has practical significance, broad market prospect, and economic value in the social development $[2,3]$.

As a newly arisen subject, fractal theory is especially suitable for analyzing complex system. Fractal theory is used in the area of fault analyzing of mechanical system as a current trend in academia. Based on the fractal theory, the vibration signals of mechanical system are analyzed, and fractal dimension is extracted as the feature information, and then the running state of the system can be analyzed not qualitatively but also quantitatively. Used with fractal theory, the faults of complex machinery system can be diagnosed, which can improve the fault identification and analysis ability. It is a practical and promising signal analyzing method for machinery devices. Based on the results of previous studies, this paper presents some research on the vibration signals 
in time-frequency domain by fractal theory. According to the simulation and experimental data, the result has shown that the analysis result varies with different vibration signals. Compared with the obvious differences in dimensions and parameters for different bearing failures, the method can be used for fault diagnosis.

\section{Empirical Mode Decomposition and Time-Frequency Transform}

In essence, the decomposition process for a signal based on EMD is a smooth processing for a signal. This decomposition will gradually decompose a complex nonlinear and nonstationary signal into different components of intrinsic mode function (IMF) and the remaining final trend. Each basic component has different characteristic scales, and the component of low-frequency limit stands for the trend and DC component of the original signal. As each intrinsic mode function stands for different local features of the original signal, different local features of the original signal can be acquired by separately analyzing the intrinsic mode function [4].

By EMD decomposition, another expression of time series can be acquired $[5,6]$ :

$$
x(t)=\sum_{i=1}^{n} C_{i}(t)+r_{n},
$$

where $C_{i}(t)$ is the intrinsic mode function and $r_{n}$ is the residual component.

The signal acquired is still a time-series signal, and the basic parameters to express signal feature are time and frequency [7]. The time characteristic scale describes the variations of signal with time, and the other signal parameter is frequency characteristic scale. The common Fourier transform can express the frequency characteristic in overall scale. The instantaneous frequency can express the frequency characteristic and time characteristic. So the instantaneous frequency can be more convincing in expressing signals $[8,9]$.

For signal $C_{i}(t)$, through Hilbert transform, $\varphi_{C_{i}}(t)$ can be acquired. Its analytic signal is

$$
Z_{C_{i}}(t)=a_{C_{i}}(t) e^{j \varphi_{C_{i}}(t)} .
$$

Instantaneous frequency $\omega_{C_{i}}$ is

$$
\omega_{C_{i}}=\varphi_{C_{i}}^{\prime}=\frac{d \varphi(t)}{d t} .
$$

It can be transformed into difference format, and the frequency can be expressed as $f_{C_{i}}(t)$ :

$$
f_{C_{i}}(t)=\lim _{\delta t \rightarrow \infty} \frac{1}{4 \pi \delta t}[\varphi(t+\delta t)-\varphi(t-\delta t)] .
$$

If sample frequency is $f_{s}$, instantaneous frequency can be defined as follows according to discrete-time signal $s(n)$ :

$$
f_{C_{i}}(n)=\frac{f_{s}}{4 \pi}[\varphi(n+1)-\varphi(n-1)] .
$$

Phase $\varphi(n)$ can be acquired by arctan function, which has the characteristic of periodicity. The value of phase is between $-\pi$ and $\pi$. Therefore, phase distribution has discontinuous characteristic on the change point. When the value of phase extends to $2 \pi, 2 \pi$ phase aberration can be produced.

Phase unwrapping algorithm is to compare the principle value of adjacent points. Consider

$$
\Delta \varphi=\varphi(n)-\varphi(n-1) .
$$

If $\Delta \varphi$ exceeds some threshold (such as $\pi$ ), then there is $2 \pi$ jump in the phase. According to positive-negative sign of $\Delta \varphi$, phase jump value can be adjusted. If $\Delta \varphi<0, \varphi^{\prime}(n)=$ $\varphi(n)+2 \pi$; if $\Delta \varphi>0, \varphi^{\prime}(n)=\varphi(n)-2 \pi$. And $\varphi^{\prime}(n)$ is the practical phase.

\section{Numerical Algorithm of Generalized Dimension in Time-Frequency Domain}

Currently, the fractal theory which is used in feature extraction of vibration signals is restricted in time domain. For the nonlinear and nonstationary signal, it is not enough to only analyze in the aspect of time domain. Combining the advantages of time domain and frequency domain, time-frequency analysis method is more suitable for the nonlinear and nonstationary vibration signal analysis of rotating machinery [10]. For time-varying characteristics of nonstationary signals, the one-dimensional signal is extended to two-dimensional time-frequency plane for calculation. The purpose is to describe the frequency components of signal or the variations of energy distribution. Using EMD decomposition for the original signal, various basic mode components and the corresponding instantaneous frequencies can be obtained with high time-frequency concentration and signal analyzing capability [11].

Multi-fractal in time-frequency domain is a method which describes the complexity of energy distribution. The traditional time domain fractal of vibration signal is based on geometric measures. By analyzing the energy distribution variations of the signals measured, Multi-fractal analysis in time-frequency domain can extract the quantitative characteristics of vibration signals. The numerical calculating method of generalized dimension of vibration signals in timefrequency domain is shown as follows.

As time series is $X_{n}$ and $X_{n}$ is carried on EMD decomposition and instantaneous frequency transform, the formula is obtained as follows:

$$
J(\omega, t)=\sum_{i=1}^{n} b_{i} A_{i}(t) e^{j \omega_{i}(t) t} .
$$

Based on the formula above, amplitude matrix $A_{U V}$ was obtained by normalization. Each point in matrix corresponds to one time and one instantaneous frequency. The squared value of the points stands for normalization energy in timefrequency domain.

Similar to sampling space division of time-domain signal [12], $\varepsilon_{j}$ stands for grid width, and $J$ stands for the number of data points of grid generation, which is called grid type. The 
number of rows and columns is separately set to $m=F / \varepsilon_{j}$, $n=T / \varepsilon_{j}$. If the grid of $m$ row and $n$ column is $m n$, the energy of grid $m n$ is $E_{m n}$, and the energy distribution probabilities of grid is

$$
P=K_{q}\left(\varepsilon_{j}\right)=\frac{E_{m n}}{\sum_{m=1}^{R} \sum_{n=1}^{P} E_{m n}}=\frac{E_{m n}}{E} .
$$

According to the above formula, $K_{q}\left(\varepsilon_{j}\right)$ can be calculated. Define $T(j)=\log \left(\varepsilon_{j}\right), S_{j}=K_{q}\left(\varepsilon_{j}\right)$, and $S(j)=-D_{q} T(j)+$ $B ; D_{q}$ is the slope of characteristic straight line (generalized dimensions) and $B$ is offset and the function can be defined as follows:

$$
f\left(D_{q}, B\right)=\sum_{j=1}^{J}\left[S(j)+D_{q} T(j)-B\right]^{2} .
$$

According to least square method, the condition of getting the minimum value for the function is [13]

$$
\frac{\partial f}{\partial D_{q}}=0, \quad \frac{\partial f}{\partial D_{q}}=0 .
$$

Then the following equation can be obtained through calculation:

$$
\begin{aligned}
D_{q}^{E}\left(\varepsilon_{j}\right) & =\frac{J \sum_{j=1}^{J} T(j) S(j)-\sum_{j=1}^{J} S(j) \sum_{j=1}^{J} T(j)}{J \sum_{j=1}^{J} T(j)^{2}-\left[\sum_{j=1}^{J} T(j)\right]^{2}} \\
& =\frac{J \sum_{j=1}^{J} K_{q}\left(\varepsilon_{j}\right) \lg \varepsilon(j)-\sum_{j=1}^{J} \lg \varepsilon(j) \sum_{j=1}^{J} K_{q}\left(\varepsilon_{j}\right)}{J \sum_{j=1}^{J}\left(K_{q}\left(\varepsilon_{j}\right)\right)^{2}-\left[\sum_{j=1}^{J} K_{q}\left(\varepsilon_{j}\right)\right]^{2}} .
\end{aligned}
$$

When $q=0$, it stands for box dimension; when $q=1$, it stands for information dimension; when $q=2$, it stands for correlation dimension. According to the rule, we can obtain $D_{3}, D_{4}, \ldots$ When different failures happen in rolling bearing, there are differences between each dimension. Particularly in the condition of high dimensions, the failure type of bearing can be judged by the differences.

\section{Chosen Principle of Grid Type $J$ and Order Parameter $q$}

Through the experimental comparison analysis for the grid generation of time-frequency signal, the change and stability of the multifractal $D_{q}$ is different when selecting different grid type $J$, which can be shown in Figure 1. How to select grid type $J$ is studied from the aspect of fractal geometry and the multifractal original definition to know the character of $D_{q}$ under different grid type $J$.

Fractal is an expression of object self-similar, so it can indicate the complexity of the measured object. While when the observation scale of the measured object is different, its complexity is not the same also. For example, when the map scale changed from big to small, the geomorphic characteristics are more and more complex. That means that

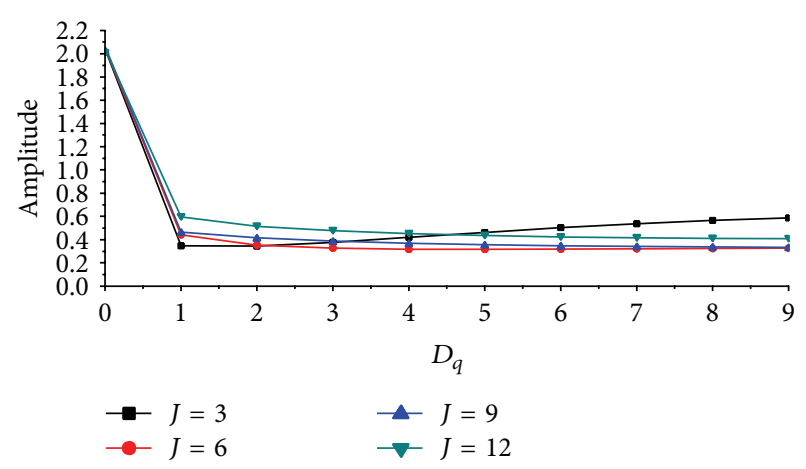

FIgURE 1: The generalized dimension curve of different grid types.

the more precise the map is divided, the higher the degree of complexity is. And that is the same for signal, which is the signal observed under high-resolution that is different from that under low resolution. And the Nyquist sampling theorem restricts the sampling rate in the process of signal acquisition.

In fractal theory, this theorem can be interpreted that the measured object should be divided into two parts at least for the self-similarity comparison. Moreover, the amount of information of every part should be same and without overlap to do the self-similarity comparison. So the largest segmentation scale of the measured object is half of itself. There are two scales of time-frequency for the signal in time and frequency domain, so the amount of information cannot be larger than a quarter of the whole information. The higher limit value of grid type $J$ can be obtained based on this theory.

For the discrete signal, it is the direct sampling value with some loss and error during the process of sampling, processing, and calculation. To eliminate the error, longer time series is used in digital signal processing. So the grid type $J$ cannot be too small; otherwise, the miscalculation may increase because of the lack of information. Therefore, how to get the lower limit value of grid type $J$ is studied.

The following is the definition of the generalized dimension, which is introduced by Renyi for the first time and found in the singular collection research by Junsheng et al. [14]:

$$
D_{q}=\frac{-\lim \lg K_{q}(\varepsilon)}{\log \varepsilon},
$$

where $K_{q}$ is called generalized entropy:

$$
K_{q}(\varepsilon)=\frac{\left\{\log \sum_{i=1}^{N}\left(P_{i}\right)^{q}\right\}}{(1-q)} .
$$

Most of the fractal dimensions in the self-similar fractal theory are included in $D_{q}$. Several properties of $D_{q}$ can be got by analyzing the formula above:

(1) $D_{q} \geq 0(\forall q \in R)$;

(2) the sum of generalized fractal dimension $D_{q}$ is monotonically decreasing with $q$ strictly.

The grid type $J$ can be verified by the two properties above. The $J$ is appropriate if it meets the two properties; 
TABLE 1: Components of two simulation signals.

\begin{tabular}{lcccc}
\hline $\begin{array}{l}\text { Simulation } \\
\text { signal }\end{array}$ & $\begin{array}{c}\text { Frequency 1 } \\
(\mathrm{Hz})\end{array}$ & Amplitude 1 & $\begin{array}{c}\text { Frequency 2 } \\
(\mathrm{Hz})\end{array}$ & Amplitude 2 \\
\hline Signal 1 & 80 & 10 & 200 & 10 \\
Signal 2 & 80 & 10 & 200 & 5 \\
\hline
\end{tabular}

otherwise, it is inappropriate. And the lower limit of $J$ can be got by this method.

From a large number of simulations and experiments, when the information distribution of the measured object is different, the lower limit of grid type $J$ is different, which has some laws as follows.

(1) The value size distribution can affect the lower limit grid type $J$ when the data distribution is the same; the wider the value distribution range is, the smaller the lower limit of grid type $J$ will be.

(2) The range and density of the data distribution can affect the lower limit of grid type $J$ when the value is the same; the wider the data distribution range and the larger the density are, the smaller the lower limit of grid type $J$ will be.

There is identifiable information in other extracted parameters in different degrees. But it does not mean that the more the characteristics are extracted, the more the useful information there will be. When the amount of characteristics reaches a certain limit, the correlation will be enhanced inevitably. But it is useless for increasing useful classified information, even the identified performance may be weakened in some cases because the important characteristics may be submerged in the unimportant characteristics.

The range of order parameter $q$ mainly affects the extracted parameters and different $q$ means that different subsets play different roles in function. Appropriate selection of $q$ can not only reflect the multifractal characteristics, but also be able to decrease correlation among different parameters as much as possible. Multi-fractal analysis divides the object to several levels with different levels through different $q$. Theoretically, the bigger the range of $q(-\infty,+\infty)$ is, the better the results will be got [15].

In the real calculation, the value of $q$ has some limits; for example, much bigger absolute value of $q$ can lead to the several times workload and overflow error will happen definitely when $q$ increases to a certain level. On the other hand, if the range of $q$ in low level, the change of $D_{q}$ is unstable but this $D_{q}$ cannot overall reflect the multifractal spectrum of the sample [16]. So the range of $q$ can be cut off at this moment when the continued increase of $q$ cannot affect the $D_{q}$ calculation result. $q$ is selected between 0 and 9 in this paper.

\section{Simulation Analysis}

Two groups of simulation signals generated by LabVIEW are compared to validate the feasibility of the theory as in Table 1.

The intensity maps are shown in Figures 2 and 3.

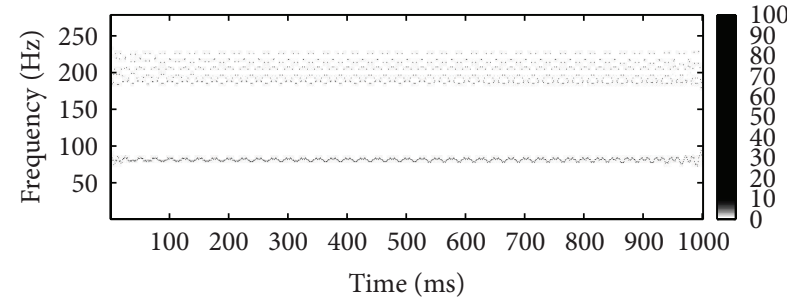

FIGURE 2: Intensity matrix of Signal 1.

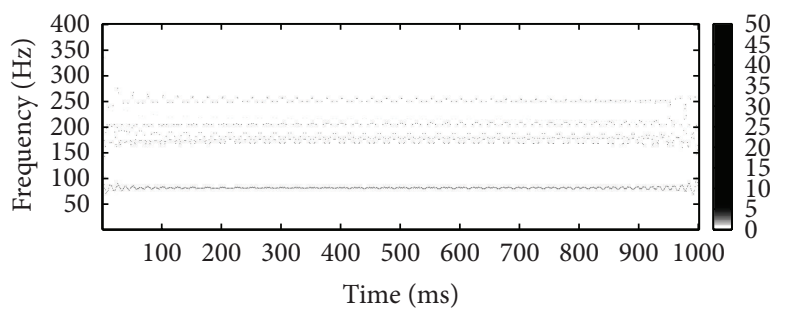

FIGURE 3: Intensity matrix of Signal 2.

TABLE 2: Components of four simulation signals.

\begin{tabular}{lccc}
\hline $\begin{array}{l}\text { Simulation } \\
\text { signal }\end{array}$ & $\begin{array}{c}\text { Frequency 1 } \\
(\mathrm{Hz})\end{array}$ & $\begin{array}{c}\text { Frequency 2 } \\
(\mathrm{Hz})\end{array}$ & $\begin{array}{c}\text { Frequency 3 } \\
(\mathrm{Hz})\end{array}$ \\
\hline Signal 1 & 50 & 100 & null \\
Signal 2 & 50 & 200 & null \\
Signal 3 & 50 & 100 & 200 \\
Signal 4 & 50 & 200 & 400 \\
\hline
\end{tabular}

From the intensity matrixes shown in Figures 2 and 3, the energy distribution of the two signal groups is roughly the same, but the energy size of them is different. The curves can be got after doing generalized dimension calculation (the grid type $J=5$ ) for the two energy intensity matrixes.

From the curves in Figure 4, the simulation Signal 2 meets the requirement when the grid type $J=5$ which has a wide amplitude distribution, while the simulation Signal 1 does not reach the lower limit of its grid type obviously. So it is explained that Rule 1 is correct. Then four simulation signals which have the same amplitude are selected to verify Rule 2 as in Table 2.

The intensity matrixes can be got after doing timefrequency analysis for the four signals shown in Figures 5, 6, 7 , and 8 .

The curves can be got after doing generalized dimension calculation for the four signals, respectively, when grid type $J=5,10,20$, and 30 shown in Figures 9, 10, 11, and 12 .

From the graphs, comparing between Signal 1 and Signal 2 , Signal 1 does not meet the requirement when $J=5$; the two curves meet the requirement of the minimum grid type when $J=10$, but the difference is still small; the two curves have some obvious differences when $J=20$; and the differences of the two curves increase and the change of them has been stable. Comparing between Signal 1 and Signal 3, the suitable $J$ of the two signals is not selected out when $J=5$; Signal 1 has 


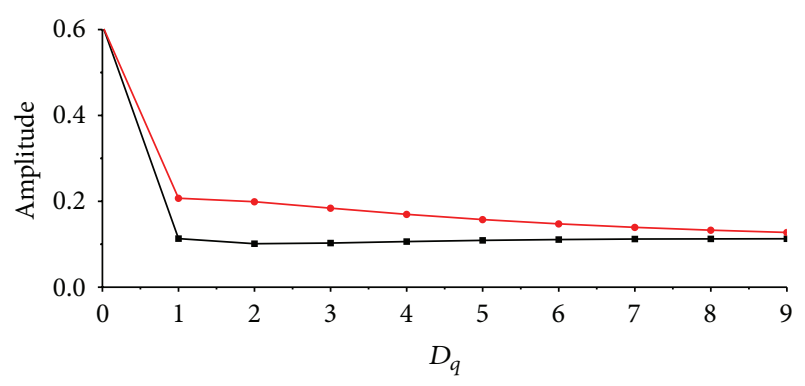

$\rightarrow$ Signal 1

$\rightarrow$ Signal 2

FIGURE 4: The generalized dimension curve of two simulation signals.

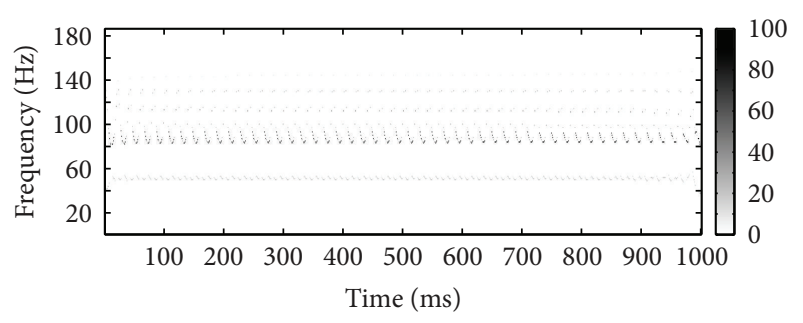

Figure 5: Intensity matrix of Signal 1.

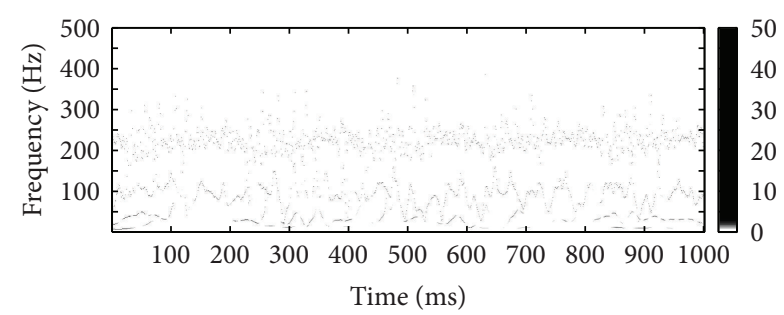

FIGURE 6: Intensity matrix of Signal 2.

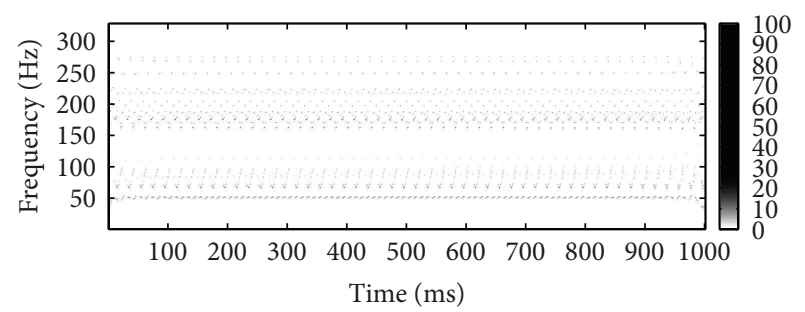

FIgURe 7: Intensity matrix of Signal 3.

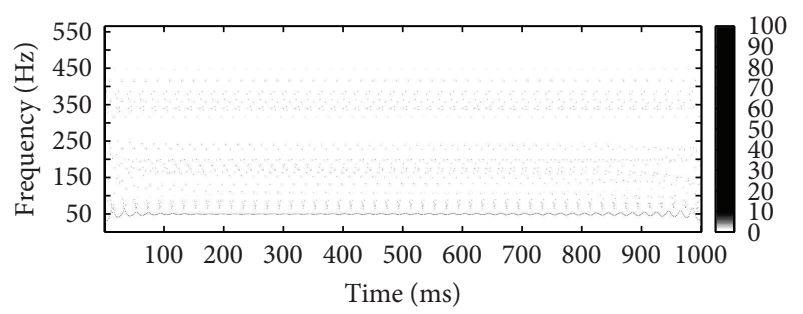

FIGURE 8: Intensity matrix of Signal 4.

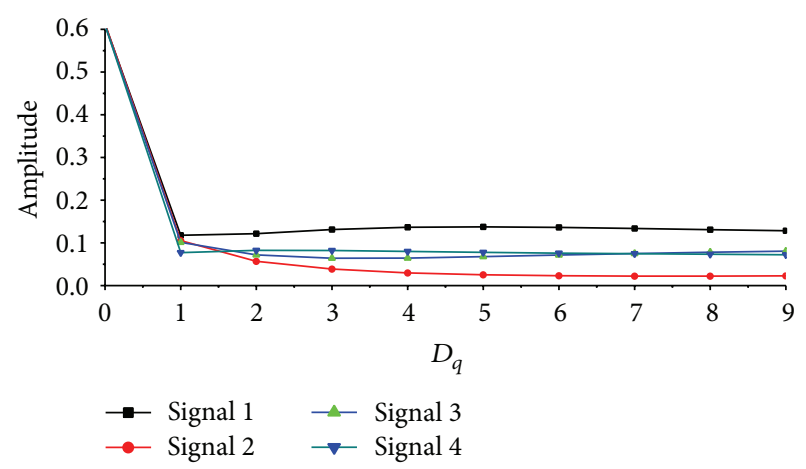

Figure 9: The generalized dimension curve $(J=5)$.

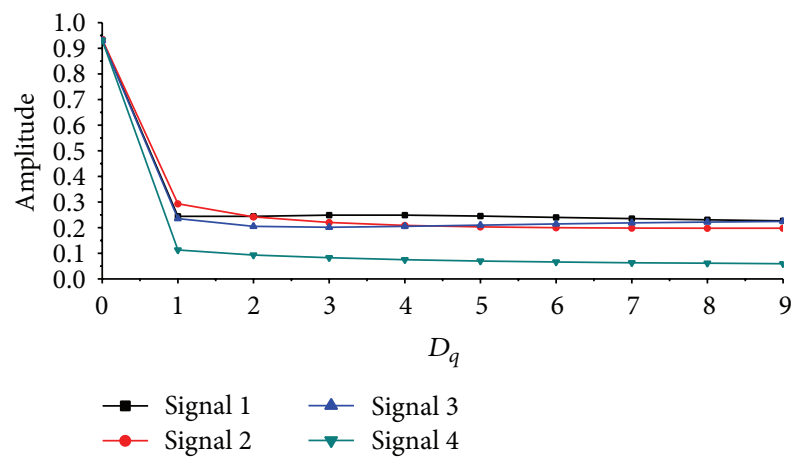

FIgURE 10: The generalized dimension curve $(J=10)$.

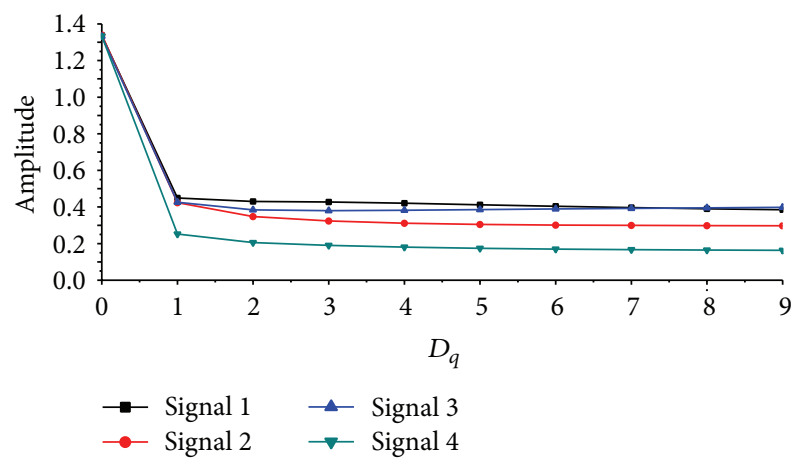

FIgURE 11: The generalized dimension curve $(J=20)$.

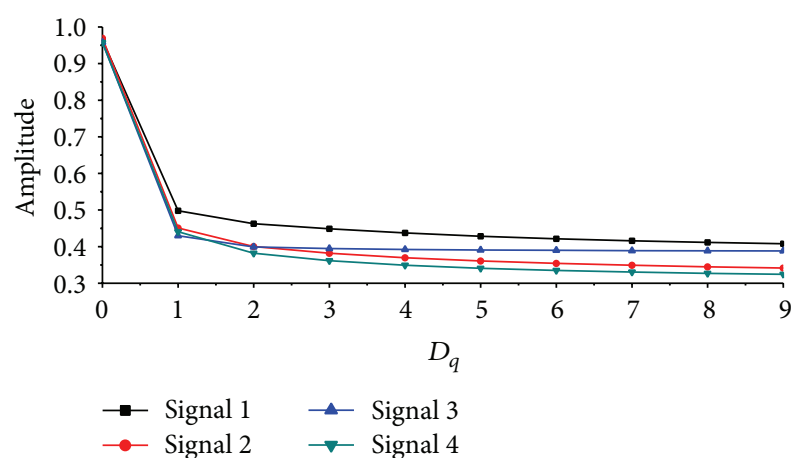

FIgURE 12: The generalized dimension curve $(J=30)$. 
TABLE 3: The bearing information of the equipment.

\begin{tabular}{lcc}
\hline Equipment number & Bearing model & Rotating speed $(\mathrm{rpm})$ \\
\hline $1 \#$ & $22320 \mathrm{CA}$ & 780 \\
$2 \#$ & $22320 \mathrm{CA}$ & 985 \\
$3 \#$ & $22320 \mathrm{CA}$ & 780 \\
$4 \#$ & $22320 \mathrm{CA}$ & 985 \\
$5 \#$ & $22320 \mathrm{CA}$ & 780 \\
\hline
\end{tabular}

reached the lower limit of the grid type when $J=10$ and 20, but Signal 2 still has not. The two signals all reach the lower limit of $J$ when $J=30$, so it is explained that Rule 2 is correct.

From the simulation experiments, the generalized dimension of different signals has good differentiation and stability while $J$ is within a certain range. The difference of generalized dimension is becoming not obvious when $J$ approaches its upper limit, and the generalized dimension $D_{q}$ is meaningless when $J$ exceeds its upper limit. When $J=130,150,150$, and 170, the calculation for the signal is shown in Figure 13 in which grid type is about 140. From the figure, curve $D_{q}$ does not satisfy the monotonous restriction and loses its significance when $J$ exceeds 140 .

Therefore, the lower limit of grid type is almost constant when the measured object is constant. To distinguish the difference of the generalized dimension when the fault happens, the grid type $J$ is selected which is higher than the lower limit until the difference can be distinguished. Meanwhile, the grid type cannot be too high, which will only increase the amount of computation but the changes of the differences are not obvious.

\section{Fault Diagnosis Example of Rolling Bearing}

From the simulation experiments, the generalized dimension can distinguish the different signals, while different signal components are generated when different faults happen to bearing, so this method can be applied in the bearing fault diagnosis. The experiment has been done in this paper to test the feasibility of the time-frequency domain dimension method further.

Doing time-frequency domain generalized dimension analysis for the real bearing measurement signal which is got by the acceleration sensor, the experimental process is shown in Figure 14.

We use the method on draught fan bearing fault detection. Table 3 is the bearing information of the equipment.

Figure 15 is the mechanical structure of the fan. The main objects are motor bearings, wheel bearings, and fan bearings. At the same time, the signal measured position is marked in the figure.

The information in detail of each measuring point is given in Table 4.

The analysis frequency of the measurement is $1 \mathrm{kHz}$, and the sampling point is $1 \mathrm{~kb}$. Data acquisition equipment information is given in Table 5 .

We obtained large fault data after long period measurement. Those data could be classified to four types: the faultfree signal, bearing cages fault signal, inner ring fault signal,
TABLE 4: Measuring point layout information.

\begin{tabular}{ccc}
\hline $\begin{array}{l}\text { Measurement } \\
\text { point }\end{array}$ & Measurement position & $\begin{array}{c}\text { Measurement } \\
\text { direction }\end{array}$ \\
\hline (1) & Belt wheel side bearing $X$ direction & Horizontal \\
1 & Belt wheel side & Vertical \\
2 & Bearing box shell $X$ direction & Horizontal \\
(2) & Bearing box shell $Y$ direction & Vertical \\
3 & & \\
4 & Fan side bearing $X$ direction & Horizontal \\
(3) & Fan side bearing $Y$ direction & Vertical \\
5 & & \\
6 & Stents $X$ direction & Horizontal \\
(4) & Stents $Y$ direction & Vertical \\
7 & & \\
8 & Motor output end bearing $X$ direction & Horizontal \\
(5) & Motor output end bearing $Y$ direction & Vertical \\
9 & & \\
10 & Fan bearing box shell side $Z$ direction & Axial \\
\hline (6) & & \\
11 & &
\end{tabular}

TABLE 5: Data acquisition equipment information.

\begin{tabular}{lcc}
\hline Equipment & Types & Model \\
\hline Sensor & ICP accelerometer & YD84D-V \\
Acquisition equipment & Data acquisition card & MPS-060602 \\
\hline
\end{tabular}

and outer ring fault signal. Those signals are selected to be analyzed.

The decomposition results of the four signals by EMD are shown in Table 6.

The first four IMFs of every signal are selected to be compared as in Figures 16, 17, 18, and 19.

From the EMD results, the four signals have different signal components. To know the characteristics of the signal components, doing the instantaneous frequency calculation and energy calculations for the four signals, respectively, the energy intensity matrix can be got as in Figures 20, 21, 22, and 23.

The energy distributions of the four signals are different at individual frequency scale. To show the difference clearly, making the grid type $J=120$ and $q$ from 0 to 9, doing generalized dimension calculation to the time-frequency domain energy matrixes for four signals, then a series of dimension values can be got as in Tables 7, 8, 9, and 10 .

The curves for the four groups of data are shown in Figure 24.

From Figure 24, the $D_{q}$ of the fault signal is bigger than the one of the normal signal, and the $D_{q}$ value difference between every fault signal and normal signal is also different. The fault type can be estimated according to the difference. So the time-frequency domain dimension method can be applied in rolling bearing fault diagnosis. 
TABLE 6: The results of EMD.

\begin{tabular}{lcccc}
\hline Signal & Fault-free & Inner ring fault & Bearing cages fault & Outer ring fault \\
\hline Number of IMFs & 4 & 5 & 6 & 6 \\
\hline
\end{tabular}

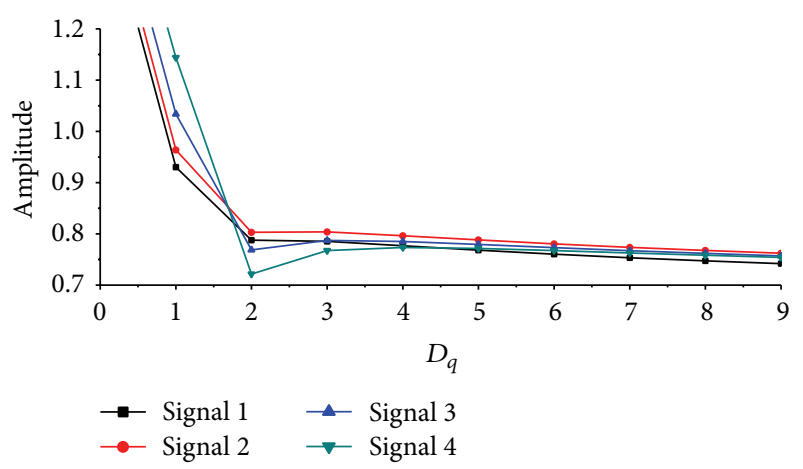

FIGURE 13: The generalized dimension curve of exceeding higher limit $J$.

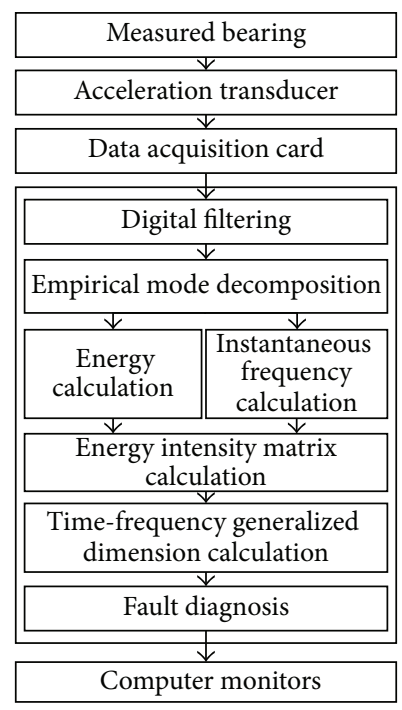

Figure 14: Experimental flow charts.

TABLE 7: The generalized dimension value of fault-free signal.

\begin{tabular}{lccccc}
\hline$q$ & 0 & 1 & 2 & 3 & 4 \\
$D_{q}$ & 2.10271 & 1.15764 & 0.68751 & 0.54359 & 0.47031 \\
$q$ & 5 & 6 & 7 & 8 & 9 \\
$D_{q}$ & 0.43111 & 0.40787 & 0.39288 & 0.38257 & 0.37513 \\
\hline
\end{tabular}

TABLE 8: The generalized dimension value of inner ring fault signal.

\begin{tabular}{lccccc}
\hline$q$ & 0 & 1 & 2 & 3 & 4 \\
$D_{q}$ & 2.10094 & 1.08670 & 0.89636 & 0.83366 & 0.78689 \\
$q$ & 5 & 6 & 7 & 8 & 9 \\
$D_{q}$ & 0.75352 & 0.72955 & 0.71193 & 0.69860 & 0.68826 \\
\hline
\end{tabular}

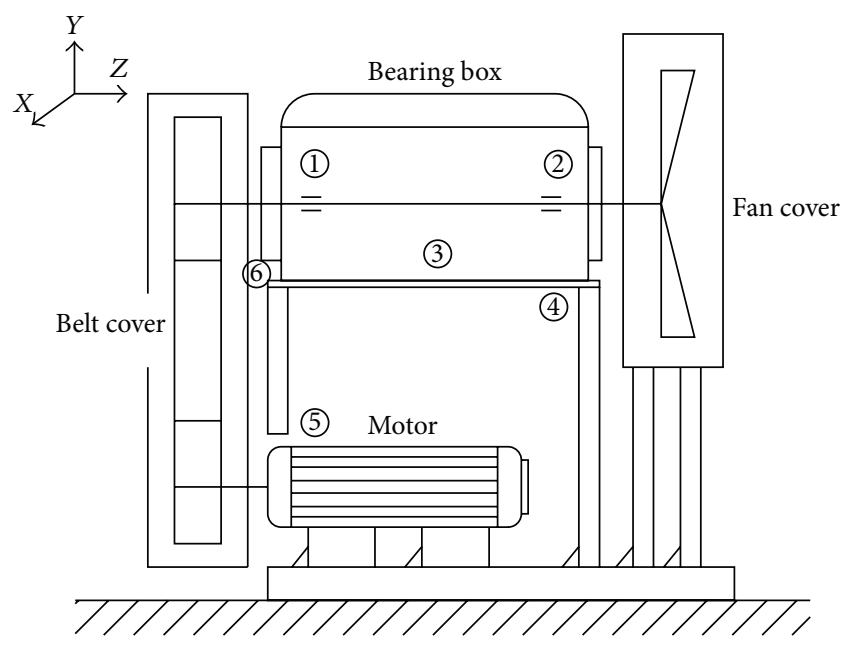

FIgURE 15: The schematic figure of equipment under test and measurement layout.

TABLE 9: The generalized dimension value of bearing cages fault signal.

\begin{tabular}{lccccc}
\hline$q$ & 0 & 1 & 2 & 3 & 4 \\
$D_{q}$ & 2.09131 & 1.08977 & 0.81367 & 0.71429 & 0.65361 \\
$q$ & 5 & 6 & 7 & 8 & 9 \\
$D_{q}$ & 0.61586 & 0.59109 & 0.57394 & 0.56149 & 0.55209 \\
\hline
\end{tabular}

TABLE 10: The generalized dimension value of outer ring fault signal.

\begin{tabular}{lccccc}
\hline$q$ & 0 & 1 & 2 & 3 & 4 \\
$D_{q}$ & 2.10112 & 1.23425 & 1.08282 & 1.04355 & 1.00652 \\
$q$ & 5 & 6 & 7 & 8 & 9 \\
$D_{q}$ & 0.97820 & 0.95706 & 0.94106 & 0.92867 & 0.91884 \\
\hline
\end{tabular}

\section{Conclusion}

Generalized dimension spectrum has been obtained after the signals are calculated by doing time-frequency domain generalized dimension method; slight difference in different singles can be distinguished based on different values of generalized dimension spectrum. And the specific algorithm and experimental analysis method have been provided. The correctness of the time-frequency domain generalized dimension method has been verified by simulation signals analysis and experimental signals analysis. The result shows that the numerical distribution of fractal dimension is different and has obvious separability under different fault modes. This indicates the correctness and feasibility of estimating the bearing fault condition qualitatively and quantitatively by time-frequency domain generalized dimension. For single or various fault types of rolling bearing, the fault mode can be 


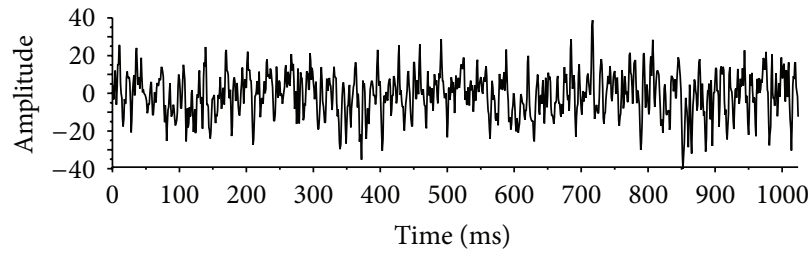

(a)

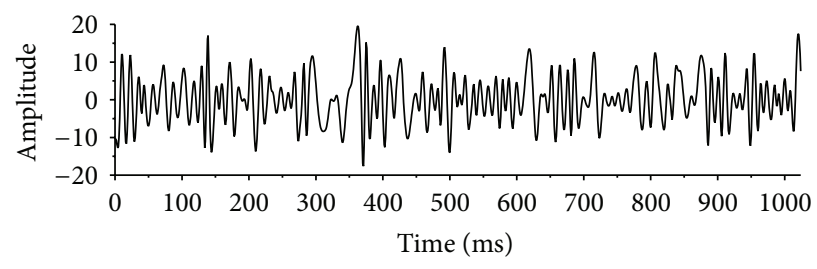

(c)

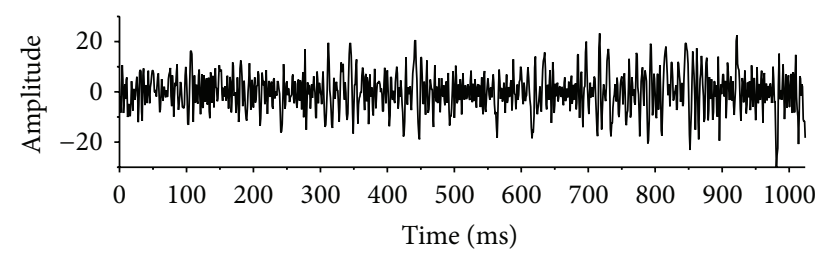

(b)

(d)

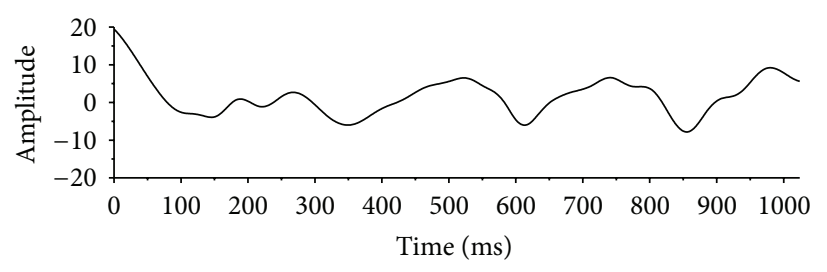

(e)

FIGURE 16: Fault-free signal and IMFs.

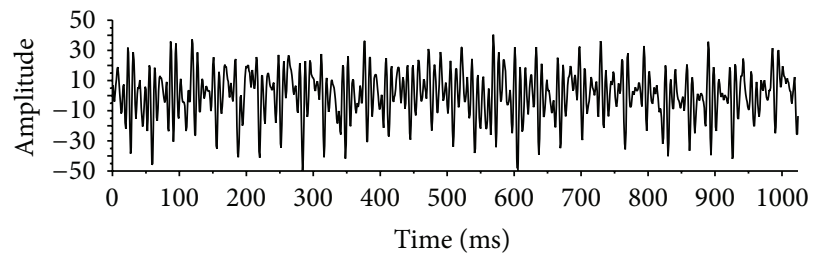

(a)

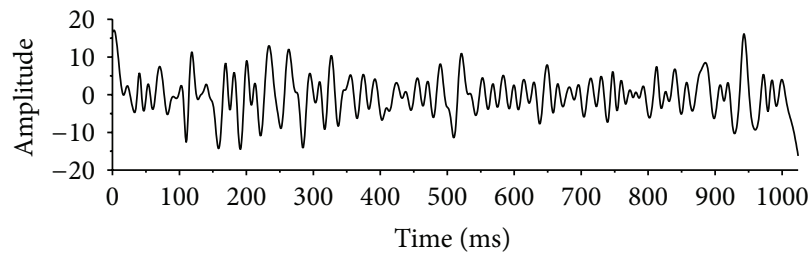

(c)

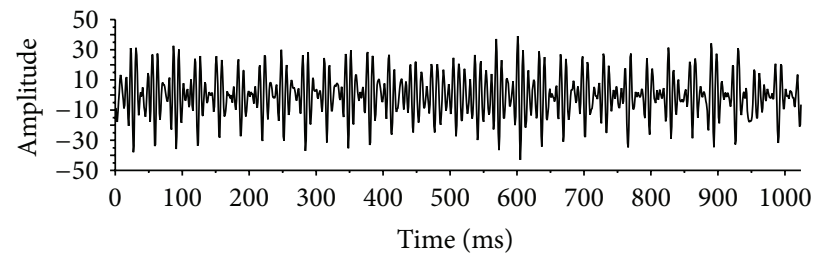

(b)

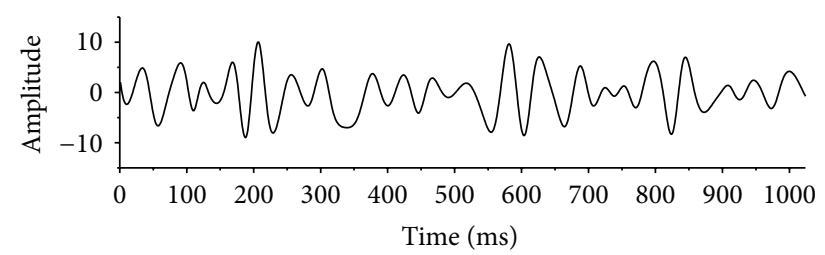

(d)

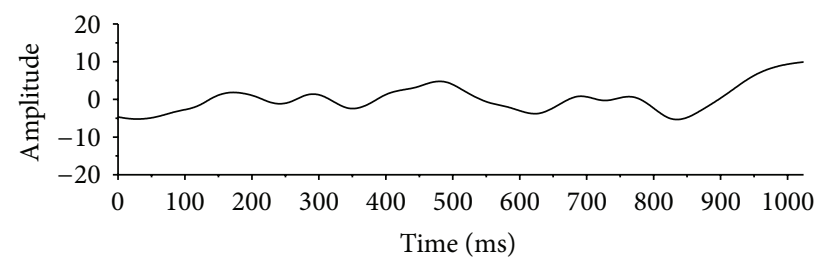

(e)

FIGURE 17: Inner ring fault signal and IMFs. 


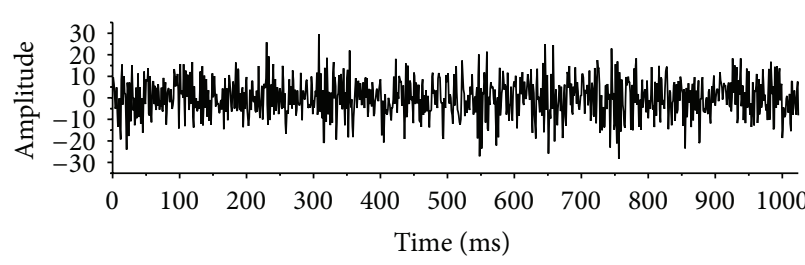

(a)

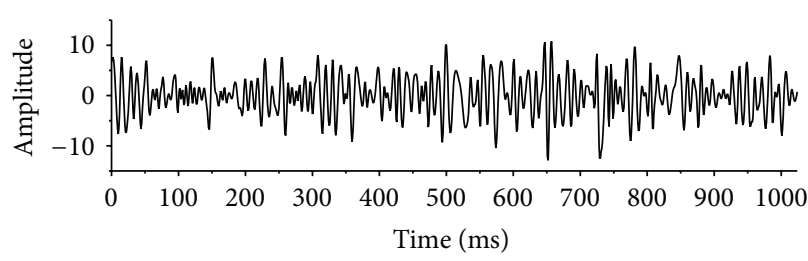

(c)

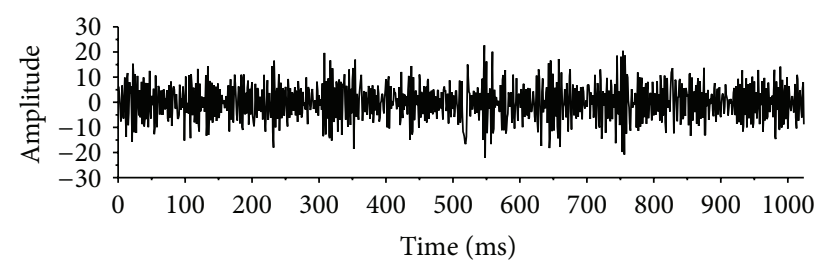

(b)

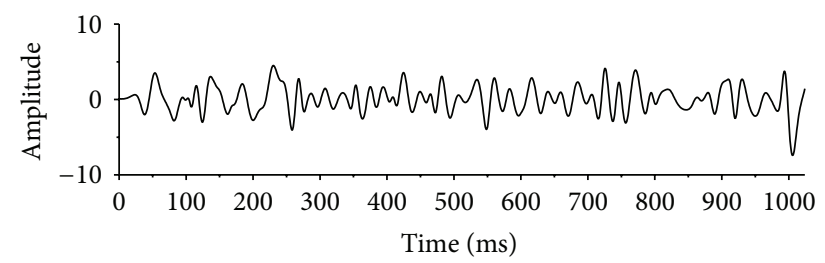

(d)

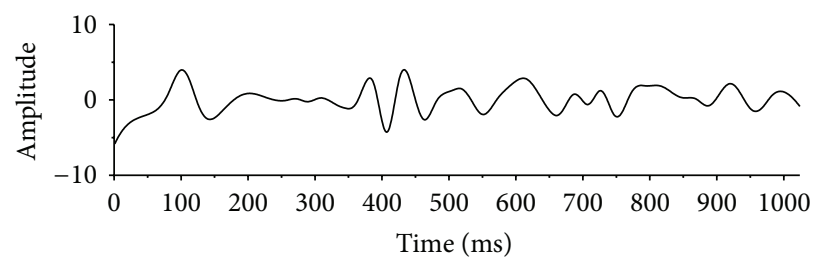

(e)

FIGURE 18: Bearing cages fault signal and IMFs.

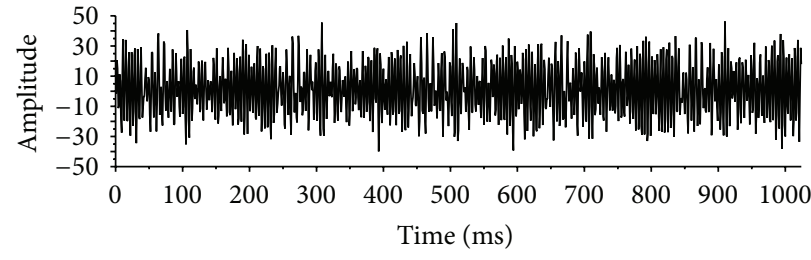

(a)

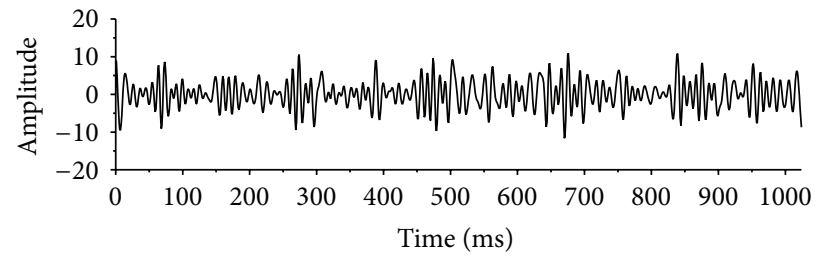

(c)

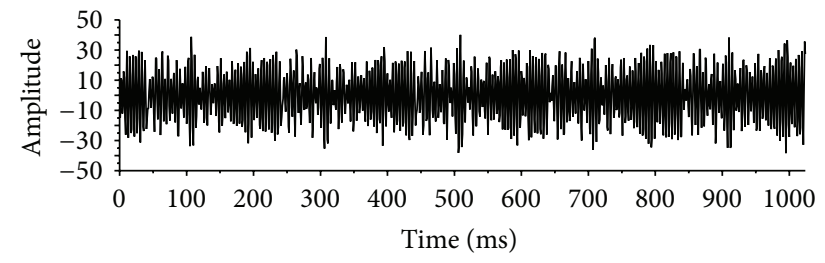

(b)

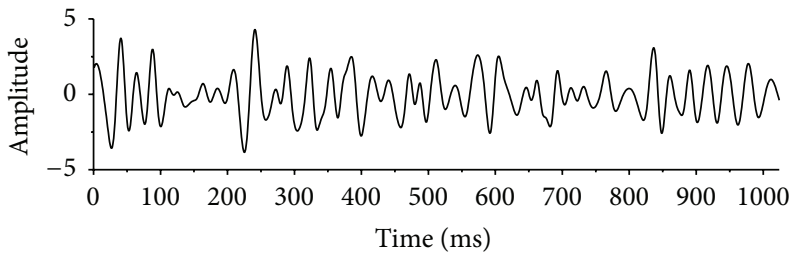

(d)

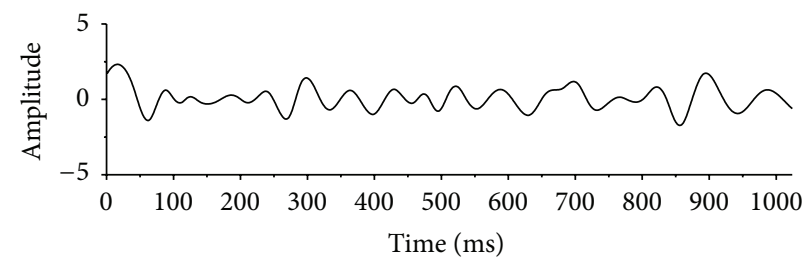

(e)

FIgURE 19: Outer ring fault signal and IMFs. 


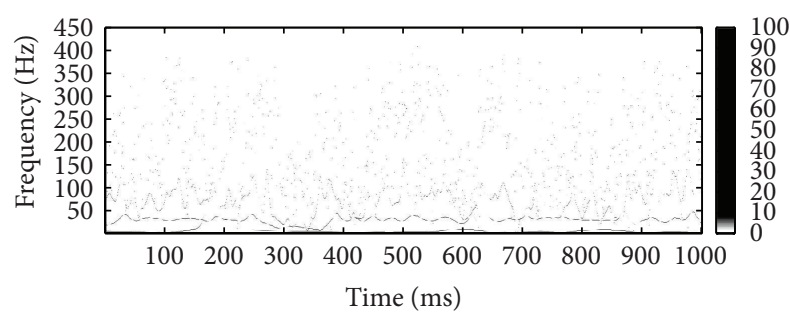

FIGURE 20: Intensity matrix of fault-free signal.

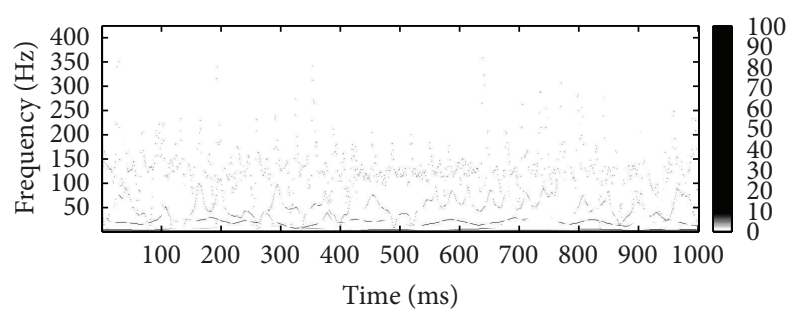

FIgURE 21: Intensity matrix of inner ring fault signal.

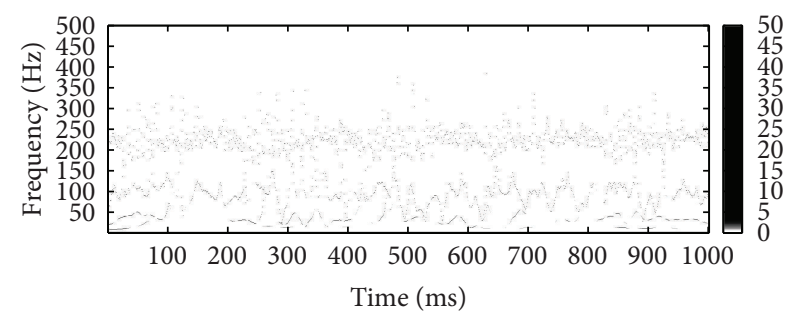

FIGURE 22: Intensity matrix of bearing cages fault signal.

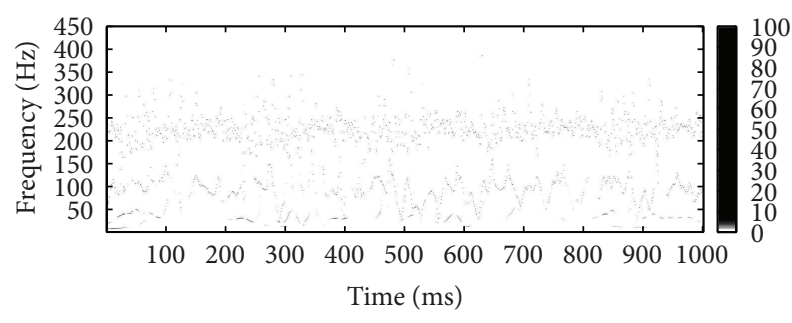

FIGURE 23: Intensity matrix of outer ring fault signal.

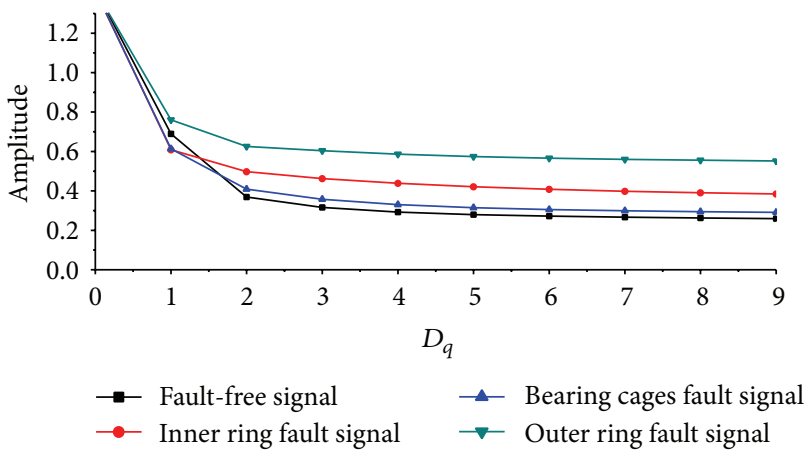

Figure 24: The generalized dimension curve of four experimental signals. confirmed by comparing the generalized fractal dimension spectrum value with the generalized fractal dimension value in the sample databases.

The working feature of bearing can be extracted by timefrequency generalized fractal dimension method. It can be applied to the diagnosis of the rolling bearing fault, and it is a simple and effective method to recognize accurately the fault models, which opens a new way for the rolling bearing fault diagnosis.

\section{Conflict of Interests}

The authors declare that there is no conflict of interests regarding the publication of this paper.

\section{Acknowledgments}

The project is supported by the National High Technology Research and Development Program (2013AA041108), the Research Foundation of Education Bureau of Liaoning Province, China (L2012166), the Foundation of State Key Laboratory (sklms2012006 and sklms2012003), and the National Natural Science Foundation of China (51475065).

\section{References}

[1] A. Muszynska and P. Goldman, "Chaotic responses of unbalanced rotor/bearing/stator systems with looseness or rubs," Chaos, Solitons and Fractals, vol. 5, no. 9, pp. 1683-1704, 1995.

[2] F. F. Ehrich, "High-order subharmonic response of high-speed rotors in bearing clearance," Journal of Vibration, Acoustics, Stress, and Reliability in Design, vol. 110, no. 1, pp. 9-16, 1988.

[3] X. Dai, Z. Jin, and X. Zhang, "Dynamic behavior of the full rotor/stop rubbing: numerical simulation and experimental verification," Journal of Sound and Vibration, vol. 251, no. 5, pp. 807-822, 2002.

[4] J. Cheng, D. Yu, J. Tang, and Y. Yang, "Application of frequency family separation method based upon EMD and local Hilbert energy spectrum method to gear fault diagnosis," Mechanism and Machine Theory, vol. 43, no. 6, pp. 712-723, 2008.

[5] F. Wu and L. Qu, "Diagnosis of subharmonic faults of large rotating machinery based on EMD," Mechanical Systems and Signal Processing, vol. 23, no. 2, pp. 467-475, 2009.

[6] Q. Gao, C. Duan, H. Fan, and Q. Meng, "Rotating machine fault diagnosis using empirical mode decomposition," Mechanical Systems and Signal Processing, vol. 22, no. 5, pp. 1072-1081, 2008.

[7] A. Papandreou-Suppappola, Ed., Applications in Time-Frequency Signal Processing, CRC Press, Boca Raton, Fla, USA, 2003.

[8] F. Hlawatsch and G. F. Boudreaux-Bartels, "Linear and quadratic time-frequency signal representations," IEEE Signal Processing Magazine, vol. 9, no. 2, pp. 21-67, 1992.

[9] I. Daubechies, "The wavelet transform, time-frequency localization and signal analysis," IEEE Transactions on Information Theory, vol. 36, no. 5, pp. 961-1005, 1990.

[10] G. Brown, "On the multi-fractal analysis of measure," Journal of Statistical Physics, vol. 66, no. 3, pp. 775-790, 1992.

[11] M. Gache, P. Flandrin, and D. Garreau, "Fractal dimension estimators for fractional Brownian motions," in Proceedings of the IEEE International Conference on Acoustics, Speech, and Signal Processing, vol. 5, pp. 3557-3560, Toronto, Canada, 1991. 
[12] K. Falconer, Fractal Geometry: Mathematical Foundations and Application, Wiley, New York, NY, USA, 1982.

[13] J. Antoni and R. B. Randall, "Differential diagnosis of gear and bearing faults," Journal of Vibration and Acoustics, Transactions of the ASME, vol. 124, no. 2, pp. 165-171, 2002.

[14] C. Junsheng, Y. Dejie, and Y. Yu, "Time-energy density analysis based on wavelet transform," NDT and E International, vol. 38, no. 7, pp. 569-572, 2005.

[15] Y. Zhou and Y. Leung, "Empirical mode decomposition and long-range correlation analysis of sunspot time series," Journal of Statistical Mechanics: Theory and Experiment, vol. 2010, Article ID P12006, 24 pages, 2010.

[16] J. Hu, J. B. Gao, and X. S. Wang, "Multiracial analysis of sunspot time series: the effects of the 11-year cycle and Fourier truncation," Journal of Statistical Mechanics, Article ID P02066, pp. 1-20, 2009. 

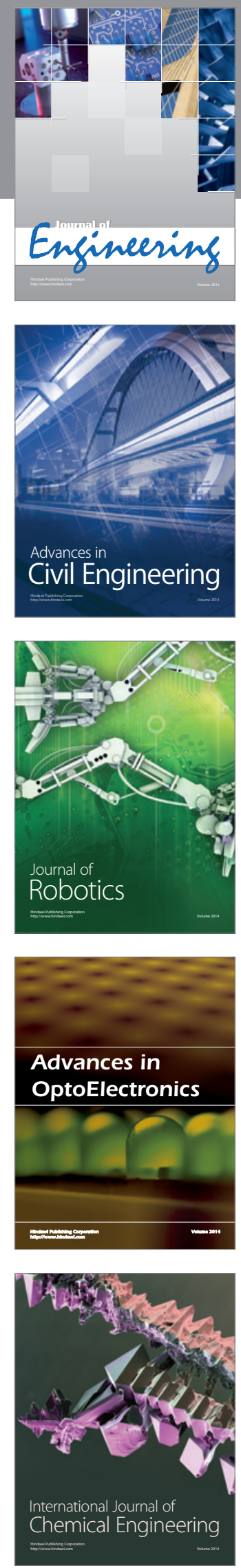

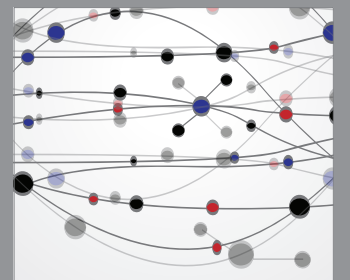

The Scientific World Journal
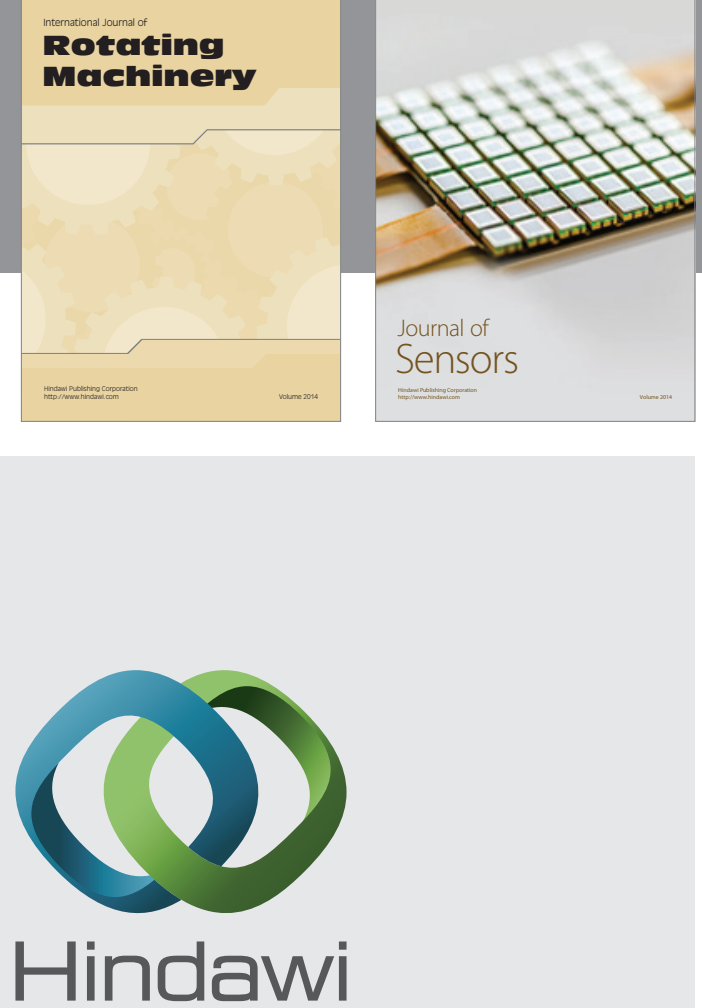

Submit your manuscripts at http://www.hindawi.com
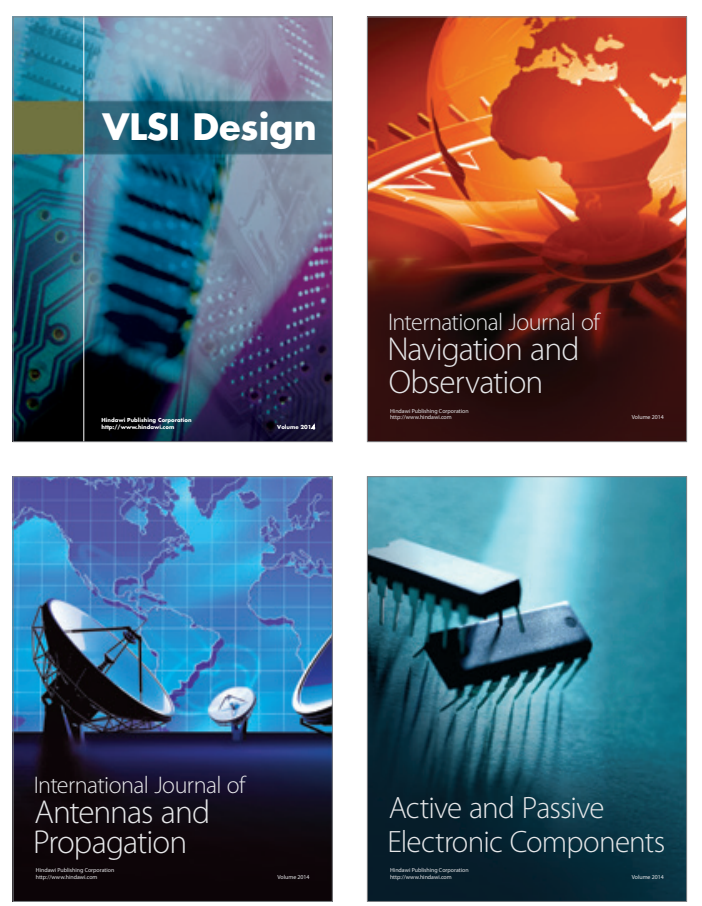
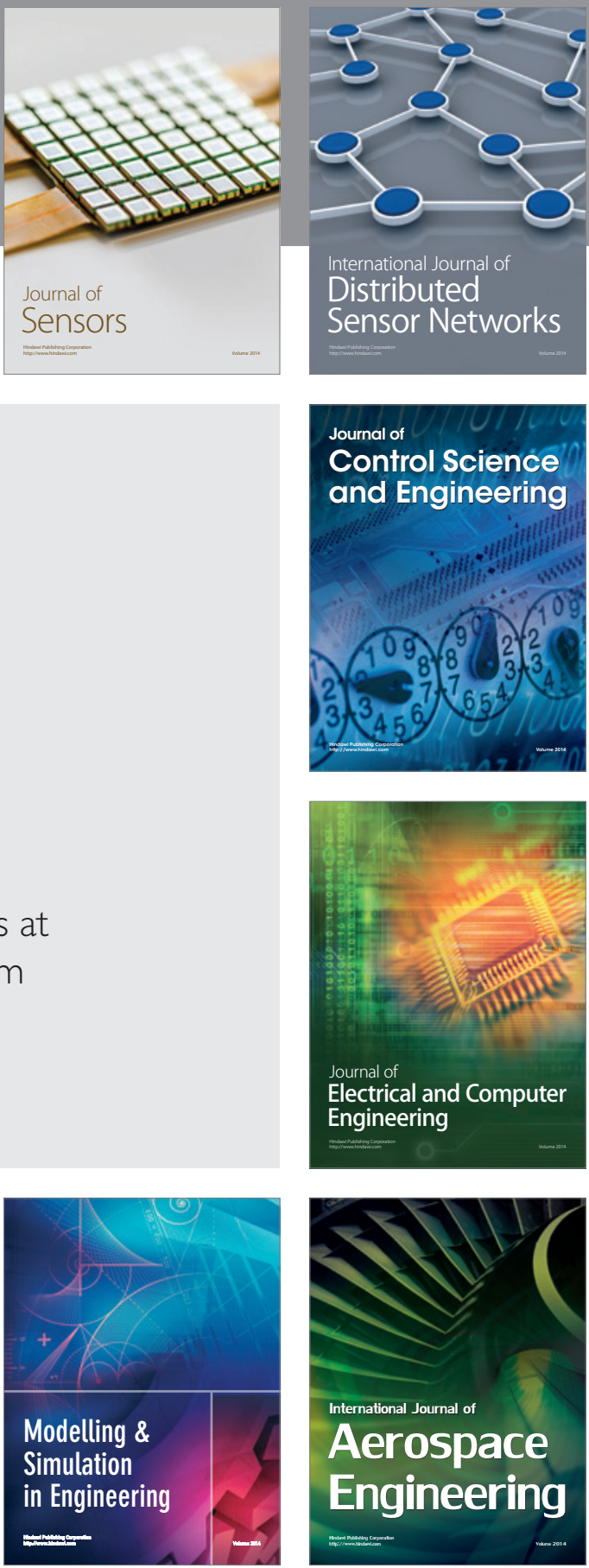

Journal of

Control Science

and Engineering
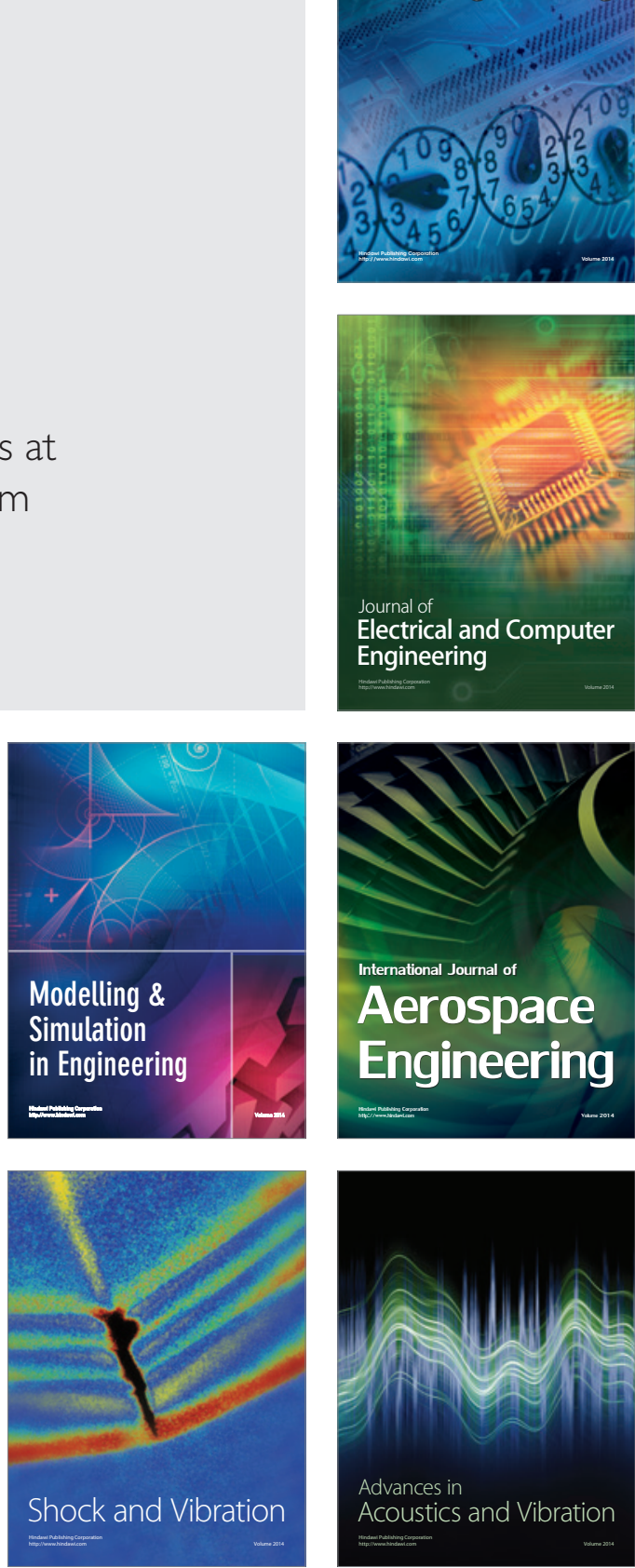